\title{
Simple analytical approximations for the gain and refractive index spectra in quantum-well lasers
}

\author{
Salvador Balle \\ Departament de Física Interdisciplinar, Instituto Mediterráneo de Estudios Avanzados, \\ IMEDEA (CSIC-UIB), E-07071 Palma de Mallorca, Spain
}

(Received 21 May 1997)

\begin{abstract}
An analytical expression for the low-temperature optical susceptibility of quantum-well semiconductor lasers is presented based on a simple parabolic band model. The optical susceptibility obtained keeps the nonlinear dependence on the carrier density, providing both a broad gain spectrum and a dispersion curve, so it can be used to analyze the dynamics of multimode devices or devices with large carrier density variations. The resulting peak gain, differential peak gain, and linewidth enhancement factor are discussed. cw operation of a single-mode laser is studied as a function of the frequency of the cavity resonance. An analytical approximation to the finite-temperature gain spectrum is also presented, although the refractive index spectrum must be determined numerically. [S1050-2947(98)07501-5]
\end{abstract}

PACS number(s): 42.55.Px, 78.66.-w

\section{INTRODUCTION}

The analysis of the static and dynamical properties of semiconductor lasers requires a knowledge of the coupling between the active semiconductor material and the optical field within the active region. In a semiclassical approach [1], which constitutes the foundation for simpler descriptions as the rate equation (RE) approximation [2], the optical field is described by means of Maxwell's equations, and its coupling to the material is described by the electrical susceptibility of the active medium. The imaginary part of the electrical susceptibility describes the energy exchange (absortion or stimulated emission) between the field and the medium, while its real part describes the dispersive effect (refractive index change) accompanying such a process [3].

This approach has been successfully undertaken for gas and solid-state lasers. In these systems, the active medium can be described in an effective way as an ensemble of atoms (or molecules) with only two levels among which stimulated emission takes place [4,5]. In this approximation, the relevant variables for describing the active medium are the population inversion between these two levels, and the corresponding nonlinear polarization. The optical Bloch equations for the evolution of these variables, together with Maxwell's equations for the evolution of the optical field, constitute the so-called two-level-model (TLM). The TLM can be reduced to a RE description when the nonlinear polarization can be adiabatically eliminated, but in general it explicitly considers the coherent coupling between the optical field and the active medium, hence allowing for large signal dynamics, multimode operation, four-wave-mixing processes, etc., which makes the TLM a very valuable tool.

Semiconductor media are conceptually similar to an ensemble of two-level atoms, though with different transition energies as defined by the electronic band structure, and more important, with different occupation of the electronic states [6-8]. These two differences make the TLM inappropriate for semiconductor media. While the gain spectrum in the TLM has a symmetric, Lorentzian shape, it is strongly asymmetric in semiconductors [6-9]. Moreover, semiconductor lasers usually exhibit a large degree of amplitudephase coupling at any operation wavelength (often described by Henry's linewidth enhancement factor $\alpha$ [10]), while the maximum gain in the TLM always occurs at the wavelength where the carrier-induced refraction index change vanishes (zero detuning); hence the large amplitude-phase coupling observed in semiconductor lasers cannot be described in the TLM unless lasing very far away from the gain peak is artificially enforced.

Models for calculating the gain and refraction index spectra from the electronic structure of the semiconductor material have been developed, some neglecting many-body effects $[6-8,11,12]$ and some taking them into account $[13-$ 15]. These microscopic theories describe individual transitions by the occupation of the initial and final electronic states, and the material polarization by superposing the contributions from each transition. A dynamical description of the lasing process then requires dealing with plenty of twolevel-like systems, coupled among them by scattering processes and by the optical field. The complexity of such a description is so high that it requires intensive numerical computation.

The complexity and high computational cost of microscopic theories has stimulated the search for simpler, analytical approximations for both the optical gain (see [16] and references therein) and the electrical susceptibility $[17,18]$ of semiconductor media. The models developed in $[17,18]$ allow one to incorporate some of the results from microscopic theories in an effective, direct way, while preserving the simplicity of the RE or TLM descriptions. The models for the electrical susceptibility introduce additional parameters in order to obtain a dynamical evolution equation for the nonlinear polarization, thus limiting the range of validity of the model. For instance, in [19] we used the model in [18] to study the dynamics of mode hopping and multimode operation of a Fabry-Pérot semiconductor laser; however, the model introduces an additional parameter $\alpha_{0}$, which limits the range of validity and introduces some artificial excess gain that has to be corrected for [19]. 
A crucial assumption in all of these approximate theories is that the carriers have relaxed to a quasiequilibrium distribution, hence the occupation probability of the electronic states is given by a Fermi-Dirac distribution with a quasiequilibrium Fermi level. Since, following a perturbation, the carrier distribution in the band only approaches a quasiequilibrium Fermi-Dirac distribution after some characteristic time $T$, the approximate theories can be used for studying dynamics only on time scales longer than $T$ [14]. Carriercarrier scattering and carrier-phonon scattering are two of the most important mechanisms driving the approach to quasiequilibrium in semiconductors. For the high carrier densities typical of semiconductor lasers, carrier-carrier scattering is the fastest relaxation mechanism, with characteristic $T$ below 1 ps [20]. Carrier-phonon scattering, which leads to the equilibration of the plasma and lattice temperatures, is a somewhat slower process with characteristic $T$ that are quite sensitive to the carrier density: for low carrier densities, carrier-phonon scattering has relaxation times $T$ of the order of $1 \mathrm{ps}$ or longer, but for the high carrier densities characteristic of QW lasers, it can be reduced to 1 ps or lower according to theoretical estimates [21]. Therefore, one can use these approximate theories when studying dynamics on time scales of several ps or longer, i.e., devices with photon lifetimes and round-trip times of several ps or longer, otherwise one would not properly take into account intracavity and propagation effects because of the breaking of the quasiequilibrium approximation for the carrier density; for shorter scale dynamics, one must resort to microscopic dynamical theories.

Nevertheless, even in the quasiequilibrium approximation, nonzero temperatures do not allow one to find approximate analytical expressions for the full optical susceptibility, but only for the material gain [16] since it just involves the imaginary part of the electrical susceptibility; the reason is that the Lorentzian shape of each transition is quite narrow as compared to the thermal energy, hence it can be approximated by a Dirac delta function. The real part of the susceptibility, which is also relevant to the lasing process since it affects the lasing frequencies through mode pulling or pushing [4], and even the mode profiles for gain-guided devices, requires a numerical evaluation for finite temperature.

In this paper I present an analytical expression for the electrical susceptibility of a quantum-well (QW) laser at low temperature. As compared to my previous work [18], all the parameters in the model are determined from the band structure, except for band-gap renormalization. The analysis is based on a simple parabolic-band approach, since the primary aim is to develop an approximation for the susceptibility that retains the key features of semiconductor media (i.e., the right dependencies of the gain and refractive index spectra on carrier density) thus allowing one to study the dynamics of multimode devices and devices with an inhomogeneous carrier density, and thereby accelerating computer simulations. The gain spectrum is highly asymmetric, and the gain peak increases sublinearly with increasing carrier density, while its position with respect to the normalized band gap experiences a blueshift due to band-filling effects. The linewdith enhancement factor is dependent on both the operation frequency and the carrier density, being different from zero at the gain peak. These characteristics make the model suitable for describing strongly multimode systems or systems where the carrier density has relatively large variations from one point to the other. A first assessment of the potentiality of the model is achieved by analyzing the $\mathrm{cw}$ operation of a single-mode laser as a function of the frequency detuning between the cavity resonance and the nominal band gap.

\section{OPTICAL RESPONSE OF SEMICONDUCTOR MEDIUM}

The optical response of a semiconductor medium is determined by the complex electrical susceptibility, which in the rotating-wave approximation with perfect $k$ conservation reads [13]

$$
\begin{aligned}
\chi_{E}(\omega)= & -\frac{i}{\varepsilon_{0}} \frac{2}{V} \sum_{l, m} \sum_{k}\left|M_{l m}(k)\right|^{2} \\
& \times \frac{f_{l}(k)-f_{m}(k)}{i\left[E_{l m}(k)-\hbar \omega\right]+\hbar \gamma(k)},
\end{aligned}
$$

where $V$ is the crystal volume, $l$ and $m$ label the bands in the crystal, $E_{l m}(k)=E_{l}(k)-E_{m}(k)$ denotes the energy difference between the electronic states, $M_{l m}(k)$ is the electric dipole element between the electronic states, $f_{l}(k)$ and $f_{m}(k)$ are the occupation probabilities of the electronic states, and the summation runs over all $k$ vectors in the first Brillouin zone and all bands. $\gamma(k)$ denotes the width of each optical transition, whose shape is assumed to be Lorentzian for the sake of simplicity. It is known that a Lorentzian line shape leads to residual absortion for photon energies below the gap, which has induced to use other line-shape functions (for a thorough discussion on the effects of using a Lorentzian line shape, see [12]). Unfortunately, line shapes other than the Lorentzian do not allow for analytical integration of the full electrical susceptibility.

For finite temperature and realistic band structures, the evaluation of $\chi_{E}(\omega)$ requires a numerical calculation; however, some insight can be gained by considering a simple parabollic band structure. For the sake of simplicity, I consider a single QW of width $W$ with only one electron and one (heavy-) hole band, although the procedure can be easily generalized to multiple electron and hole bands. The situation considered here is approximately the one occurring in a strained, narrow QW, where only one electron and one heavy-hole band are active until large carrier densities are injected into the QW. In the approximation of parabolic bands, $E_{c}=E_{t}+\left(\hbar^{2} k^{2} / 2 m_{c}\right)$ and $E_{v}=-\left(\hbar^{2} k^{2} / 2 m_{v}\right)$, where $m_{c}\left(m_{v}\right)$ is the conduction-band (valence-band) effective mass and $E_{t}$ is the energy difference between the conduction and valence band at $k=0$. Rewriting $\chi_{E}(\omega)$ in terms of the electron and hole distribution functions, $f_{e}=f_{c}$ and $f_{h}=1$ $-f_{v}$, with $E_{h}=-E_{v}$, and assuming that $M_{c v}(k)=M$ and $\gamma(k)=\gamma$ are independent of $k$, one finds

$$
\begin{aligned}
\chi(\omega) & =\frac{|M|^{2}}{\varepsilon_{0}} \frac{2}{V} \sum_{k} \frac{f_{e}(k)+f_{h}(k)-1}{\hbar \omega-E_{c v}(k)+i \hbar \gamma} \\
& \equiv \chi_{e}(\omega)+\chi_{h}(\omega)-\chi_{b}(\omega),
\end{aligned}
$$

where 


$$
\chi_{e(h)}(\omega)=\frac{|M|^{2}}{\varepsilon_{0}} \frac{2}{V} \sum_{k} \frac{f_{e(h)}(k)}{\hbar \omega-E_{c v}(k)+i \hbar \gamma},
$$

and analogously for the last term, $\chi_{b}(\omega)$, except for the distribution function in the numerator. $\chi_{b}(\omega)$ can be analytically calculated, yielding

$$
\chi_{b}(\omega)=-\frac{m|M|^{2}}{W \pi \varepsilon_{0} \hbar^{2}} \ln \left(1-\frac{\hbar k_{m}^{2}}{2 m z}\right),
$$

with $z=\omega-E_{t} / \hbar+i \gamma, m=\left(m_{c}^{-1}+m_{v}^{-1}\right)^{-1}$ being the reduced mass of the electron-hole pair, and $k_{m}$ the maximum wave vector contained in the first Brillouin zone.

In general, $\chi_{e}(\omega)$ and $\chi_{h}(\omega)$ cannot be evaluated analytically, since they involve the carrier distribution in the bands. In the presence of an optical field, the carrier distribution in the band is usually not known until scattering processes have driven the system to the quasiequilibrium state. As commented above, the high carrier densities typical of QW lasers imply that carrier-carrier scattering (with $T \sim 1 \mathrm{ps}$ ) are dominant, although the slower carrier-phonon scattering mechanism is responsible for the slower thermal effects observed in many lasers. In this limit, the electron and hole densities are described by their corresponding quasiequilibrium Fermi levels, $F_{e}$ and $F_{h}$, respectively. Nevertheless, even in the quasiequilibrium approximation the electrical susceptibility cannot be calculated analytically unless one considers very low temperature, where the Fermi-Dirac distributions closely resemble step functions. In this case, one obtains

$$
\chi_{e(h)}(\omega)=-\frac{m|M|^{2}}{W \pi \varepsilon_{0} \hbar^{2}} \ln \left(1-\frac{\pi W \hbar N_{e(h)}}{m z}\right),
$$

with $\quad N_{e}=\left(m_{c} / W \pi \hbar^{2}\right)\left(F_{e}-E_{t}\right) \Theta\left(F_{e}-E_{t}\right) \quad$ and $\quad N_{h}$ $=\left(m_{c} / W \pi \hbar^{2}\right) F_{h} \Theta\left(F_{h}\right)$ being the electron and hole densities, respectively, and $\Theta(x)$ the step function. Assuming charge neutrality within the QW, $N_{e}=N_{h}=N$, the electrical susceptibility can then be written as

$$
\chi(\omega, N)=-\chi_{0}\left[2 \ln \left(1-\frac{D}{u+i}\right)-\ln \left(1-\frac{b}{u+i}\right)\right],
$$

where I have defined

$$
\begin{gathered}
\chi_{0}=\frac{m|M|^{2}}{W \pi \varepsilon_{0} \hbar^{2}}, \quad D=\frac{\pi W \hbar}{m \gamma} N \equiv \frac{N}{N_{t}}, \\
u=\frac{\operatorname{Re}(z)}{\gamma}=\frac{\left(\omega-E_{t} / \hbar\right)}{\gamma}, \quad b=\frac{\hbar k_{m}^{2}}{2 m \gamma} .
\end{gathered}
$$

In these expressions, band-gap renormalization effects due to the screened Coulomb interaction between electrons and holes have not been taken into account. However, they can be effectively implemented in a rigid band approximation by considering that the transition energy $E_{t}$ corresponds to the renormalized transition energy. The functional form of the band gap shrinkage with carrier density is still an open question, and linear [22], square-root [23], and cubic-root [24] dependencies have been proposed. This last form seems to be closest to experimental results [24], henceforth I will consider that $E_{t}=E_{t}^{0}-s N^{1 / 3}$, where $E_{t}^{0}$ is the transition en- ergy when no carriers are injected into the QW and $s$ describes the band-gap shrinkage as a function of carrier density, which depends on both the material forming the active layer and the operating conditions. Thus, I accordingly redefine

$$
u=\frac{\omega-E_{t}^{0} / \hbar}{\gamma}+\sigma D^{1 / 3} \equiv \frac{\omega-\omega_{0}}{\gamma}+\sigma D^{1 / 3},
$$

with $\omega_{0}=E_{t}^{0} / \hbar$ being the normalized nominal transition frequency, and $\sigma=s N_{t}^{1 / 3} /(\hbar \gamma)$ describing the band-gap shrinkage.

The above expression (6) for the electrical susceptibility is remarkably simple, allowing one to obtain several exact results, which are next discussed.

\section{A. Material gain spectrum}

The material gain spectrum is determined from the imaginary part of the electrical susceptibility as

$$
g(\omega, N)=-\frac{\omega}{c \bar{n}} \operatorname{Im}[\chi(\omega, N)] .
$$

The imaginary part of the electrical susceptibility can be written as

$$
\begin{aligned}
\operatorname{Im}[\chi(\omega, N)]= & -\chi_{0}[2 \arg (u-D+i)-\arg (u+i) \\
& -\arg (u-b+i)] \\
\approx & -\chi_{0}\left[\arctan (u)-2 \arctan (u-D)-\frac{\pi}{2}\right],
\end{aligned}
$$

where $\arg (z)$ is the polar angle of the complex number $z$. The approximation comes from the fact that $b \gg 1$ and $b$ $\gg u$, since the frequencies of interest are not too far away from $\omega_{0}$.

Figure 1 shows $\operatorname{Im}[\chi(\omega, N)]$ as a function of $\omega$ for different values of $N$ and two different values of the band-gap renormalization parameter, $\sigma=0.2$ (solid lines) and $\sigma=2$ (dashed lines). It can be seen that the gain value is independent of $\sigma$, but not the location of the gain peak. As already mentioned, some residual absorption below the band gap is observed due to the slowly decaying tails of the Lorentzian line shape that I have considered for the electronic transitions. For high frequency $-\operatorname{Im}[\chi(\omega, N)]$ saturates at the value $-\pi \chi_{0}$, because only one transition has been considered. As the carrier density increases, $-\operatorname{Im}[\chi(\omega, N)]$ develops a peak whose height and full width at half maximum increase; also, the position of the maximum experiences a blueshift for increasing carrier density. The peak value becomes positive for $N>N_{t}$, hence $N_{t}=m \gamma /(\pi W \hbar)$ is the transparency carrier density; the transparency frequency is $\omega=\omega_{0}-\sigma \gamma$, which is the frequency corresponding to the renormalized transition. The high-frequency wing of the peak decays quite fast, the reason being that, in the lowtemperature limit considered, the carrier distribution within the band vanishes above the quasi-Fermi level; as discussed later in this section, for higher temperatures, the states above the quasi-Fermi level have nonvanishing occupation prob- 


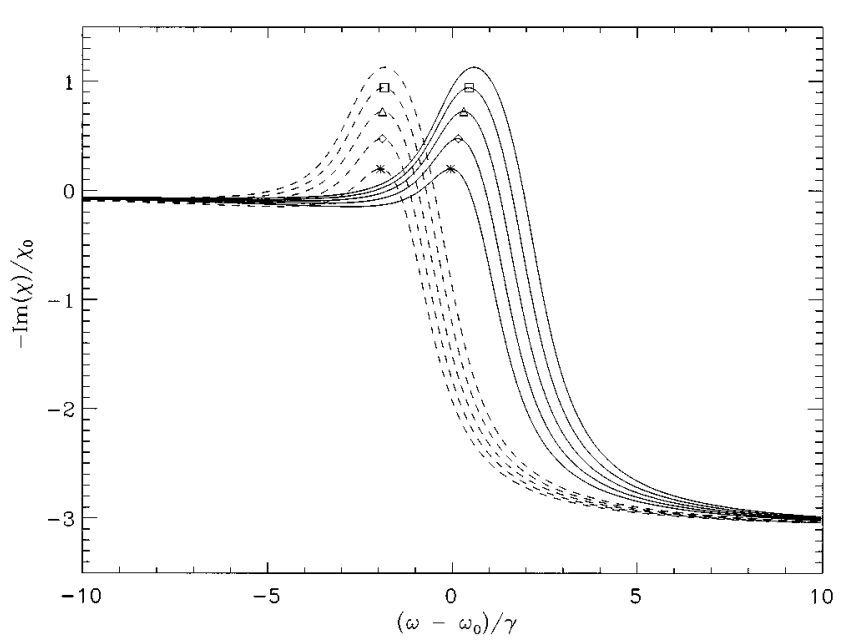

FIG. 1. Normalized imaginary part of the susceptibility as a function of the normalized frequency deviation from the nominal band-gap frequency, $\left(\omega-\omega_{0}\right) / \gamma$, for increasing carrier densities $N / N_{t}=1.2$ (star), 1.5 (diamond), 1.8 (triangle), 2.1 (square), and 2.4 (no symbol). Solid lines correspond to $\sigma=0.2$, while dashed lines correspond to $\sigma=2$.

ability, thus reducing the absorption and leading to a smoother decay for high frequencies.

From Eq. (8) it is simple to show that positive gain can only be achieved for $u \leqslant D$, which is the Bernard-Duraffourg condition at zero temperature. Actually, the frequency domain where one has positive gain (for a fixed carrier density) is given by

$$
-\sqrt{D^{2}-1} \leqslant u \leqslant \sqrt{D^{2}-1}
$$

Obviously, there is positive gain only for $D>1$, the transparency frequency being $u=0$, in agreement with the numerical results.

The frequency where the peak occurs is given by

$$
u_{p}=-D+\sqrt{2 D^{2}-1} \Rightarrow \frac{\omega_{p}-\omega_{0}}{\gamma}=-D+\sqrt{2 D^{2}-1}-\sigma D^{1 / 3}
$$

The first two contributions represent the blueshift of the peak position due to carrier band filling, partially compensated by the third term, which describes the redshift of the peak frequency due to the band-gap shrinkage. It is worth noticing that the gain peak develops only for carrier densities larger than $N_{t} / \sqrt{2}$; below this point, the Lorentzian broadening smears out the contribution of the carrier density in the steplike absorption spectrum.

Since in most cases the region of positive gain is restricted to a narrow interval in the vicinity of the nominal transition frequency, we can approximate

$$
g(\omega, N)=g_{0}\left[\arctan (u)-2 \arctan (u-D)-\frac{\pi}{2}\right]
$$

with $g_{0}=\chi_{0} E_{t}^{0} / \hbar c \bar{n}$ being the material gain coefficient. By using Eq. (10), we can determine the peak gain, which reads
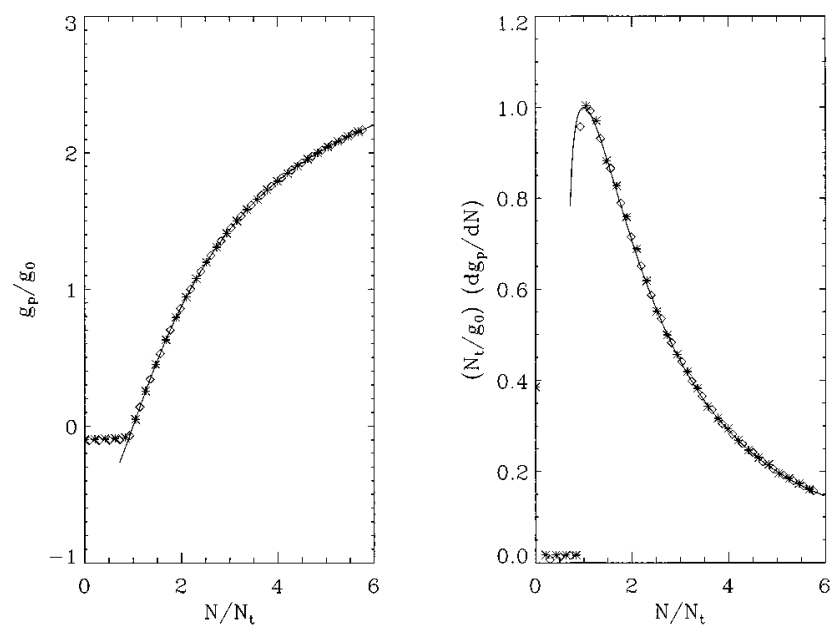

FIG. 2. Normalized peak gain (left panel) and differential peak gain (right panel) as a function of the normalized carrier density $N / N_{t}$, for $\sigma=0.2$ (stars) and $\sigma=2$ (diamonds). The symbols are obtained numerically from the susceptibility as given by Eq. (6), while the solid lines are found from Eqs. (12) (left) and (13) (right).

$$
\begin{aligned}
g_{p}= & g_{0}\left[\arctan \left(-D+\sqrt{2 D^{2}-1}\right)-2 \arctan (-2 D\right. \\
& \left.\left.+\sqrt{2 D^{2}-1}\right)-\frac{\pi}{2}\right]
\end{aligned}
$$

and the differential peak gain,

$$
\frac{d g_{p}}{d N}=\frac{g_{0}}{N_{t}} \frac{1}{D\left(3 D-2 \sqrt{2 D^{2}-1}\right)} .
$$

The peak gain and differential peak gain are shown in Fig. 2 , and-as expected-they do not depend on $\sigma$. It is clear that the gain peak grows sublinearly with carrier density, with a saturation value $g_{p}=\pi g_{0}$ that corresponds to complete inversion (again, this is a consequence of having taken into account only one electron and one hole band). It can be seen (left panel) that the gain peak does not correspond to maximum gain (in fact, minimum absorption) for carrier densities below transparency. In this case, the almost constant gain peak value corresponds to the residual absorption that occurs below the transition energy. This is a consequence of the already commented on smearing out of the gain due to Lorentzian broadening. The differential peak gain rapidly decreases from its maximum value $g_{0} / N_{t}$ at transparency $(D=1)$ to only $20 \%$ at $D=5$.

The shape of both curves is very similar to those obtained experimentally [25-27] or calculated from realistic band structures [8], although these results correspond to ambient temperature and real band structures. However, the hightemperature values for $N_{t}$ are quite higher and those for $g_{0}$ quite lower than those obtained from Eq. (6). Nevertheless, if one lets $N_{t}$ and $g_{0}$ be adjustable parameters, the results in [8] can be nicely fitted to the above functional forms with better agreement for systems with a narrow, strained QW, which only possess one electron and one hole band active until large carrier densities are injected. 
An analytical approximation for the gain at ambient temperatures can be developed [16] by considering that the individual transitions possess a linewidth much narrower than the steplike decrease in the Fermi-Dirac distribution functions (typically, the width of individual transitions is $\hbar \gamma$ $\sim 6 \mathrm{meV}$, while at ambient temperature, $\beta^{-1}=k_{B} T \sim 25$ $\mathrm{meV}$ ). From Eq. (2) we have

$$
\begin{aligned}
-\operatorname{Im}[\chi(\omega, N)] \approx & \frac{|M|^{2}}{\varepsilon_{0}} \frac{2}{V} \sum_{k}\left[f_{e}(k)+f_{h}(k)-1\right] \\
& \times \frac{\hbar \gamma}{\left[\hbar \omega-E_{c v}(k)\right]^{2}+(\hbar \gamma)^{2}},
\end{aligned}
$$

and assuming that the Fermi-Dirac distributions remain approximately constant within $\hbar \gamma$ and that the frequency is in the vicinity of the nominal transition frequency, we find

$$
\begin{aligned}
g(\omega, N) \approx & g_{0}\left[\frac{\pi}{2}+\arctan (u)\right] \\
& \times\left(\frac{e^{a_{c} D}-1}{e^{a_{c} D}-1+e^{a_{c} u}}+\frac{e^{a_{v} D}-1}{e^{a_{v} D}-1+e^{a_{v} u}}-1\right),
\end{aligned}
$$

where $a_{c(v)} \equiv \beta \hbar \gamma m / m_{c(v)}$, and $\pi / 2+\arctan (u)$ is the (broadened) reduced density of states in the QW under the assumption of a single electron and hole band. Since Eq. (15) can be rewritten as a combination of hyperbolic tangents, which have exactly the same first two terms in a Taylor expansion as an arctangent, Eqs. (11) and (15) can be made almost identical in some frequency interval (around the gain peak, say) by proper choice of the scaling parameters, and the peak gain and the differential gain at the peak can be made virtually identical for the two approximations. The main differences among the two are noticeable on the highfrequency wing, where the slowly decaying Fermi-Dirac distribution reduces the absorption.

\section{B. Carrier-induced dispersion and linewidth enhancement factor}

Another important characteristic of semiconductor lasers is the strong dispersive effect accompanying material gain, which leads to a high degree of AM-FM coupling, usually described by means of Henry's linewidth enhancement factor, $\alpha$ [10]. The linewidth enhancement factor describes the changes in the refractive index of the system that occur associated to changes in the gain or absorption as the carrier density varies. It is rather often taken to be constant, despite the existing evidence of its dependence on both frequency and carrier density $[14,16,25,27,28]$.

Refractive index changes influence lasing action in semiconductor lasers in two different ways: on one hand, they induce mode pulling or pushing through the phase change over one cavity round trip [4]; on the other, the waveguide structure of the semiconductor laser may be substantially modified, especially for weakly index-guided and gainguided devices, thus changing the optical confinement factor and the modal gain spectrum [29]. The refractive index change is associated with the real part of the electrical sus- ceptibility (see Fig. 3), which can be written as

$$
\begin{aligned}
\operatorname{Re}[\chi(\omega, N)] & =\chi_{0} \ln \left[\frac{\sqrt{u^{2}+1} \sqrt{(u-b)^{2}+1}}{(u-D)^{2}+1}\right] \\
& \approx \chi_{0} \ln \left[b \sqrt{\frac{u^{2}+1}{(u-D)^{2}+1}}\right],
\end{aligned}
$$

where the approximation comes from the fact that lasing occurs for frequencies close to the nominal one, so we always have $b \gg|u|$. As a consequence, the main role of $b$ is simply to set the background value of the refraction index.

From Fig. 3 we can see that as the carrier density increases, the refractive index decreases for low frequencies while for high frequencies it increases. The bump in all curves corresponds to the frequency region just above that of positive gain, and it experiences a blueshift as band filling occurs. It is worth noticing that the shape of the real part of the susceptibility is almost independent on the degree of band-gap shrinkage, but not the value of the refractive index change for fixed frequency. For large band-gap renormalization, the curves for different carrier densities cluster tightly together, since the band-filling contribution is more strongly compensated by band-gap renormalization, thus leading to smaller index variations (for fixed wavelength) as compared to the case with small band-gap renormalization. As a consequence, one might expect a reduction in the value of $\alpha$ for increasing band-gap shrinkage, in agreement with [14].

The linewidth enhancement factor $\alpha$ can be determined as

$$
\alpha=\frac{\operatorname{Re}(\partial \chi / \partial N)}{\operatorname{Im}(\partial \chi / \partial N)}
$$

which is now a function of both frequency and carrier density (see Fig. 4). For high carrier densities, $\alpha$ develops a bump that has been observed in some cases [28]. As already commented, it turns out that $\alpha$ is extremely sensitive to band-gap renormalization, in agreement with previous work [14]. However, it must be noted that $\alpha$ is rather sensitive not

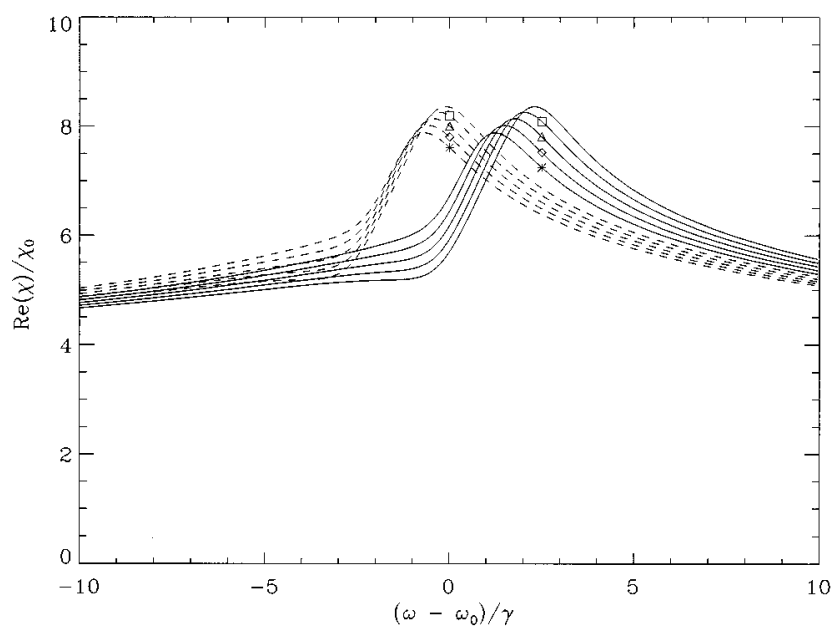

FIG. 3. Normalized real part of the susceptibility as a function of the normalized frequency deviation from the nominal band-gap frequency, $\left(\omega-\omega_{0}\right) / \gamma$ for the same carrier densities and $\sigma$ values as in Fig. 1. 


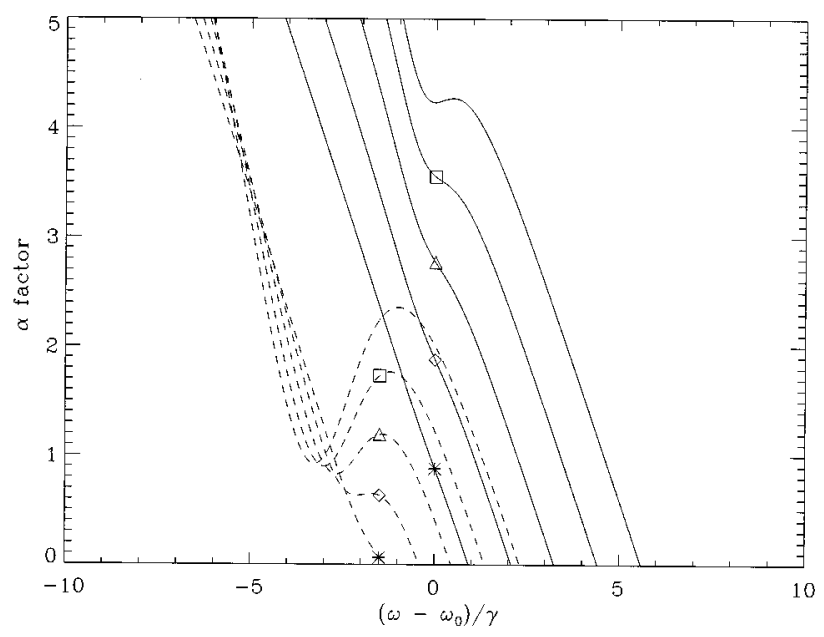

FIG. 4. Linewidth enhancement factor $\alpha$ as a function of the normalized frequency deviation from the nominal band-gap frequency, $\left(\omega-\omega_{0}\right) / \gamma$, for carrier densities $N / N_{t}=1.17$ (star), 2.37 (diamond), 3.57 (triangle), 4.77 (square), and 5.97 (no symbol). Solid lines correspond to $\sigma=0.2$, while dashed lines correspond to $\sigma=2$.

only to the amount of band-gap renormalization, but also to its functional dependence on the carrier density. The reason is that, when band-gap shrinkage is taken into account, one has that

$$
\frac{\partial}{\partial N}=\frac{1}{N_{t}}\left(\frac{\partial}{\partial D}+\frac{\partial u}{\partial D} \frac{\partial}{\partial u}\right)
$$

so that the variation of band-gap shrinkage with increasing carrier density comes into play.

The linewidth enhancement factor at the frequency of the gain peak is given by

$$
\alpha_{p}=2 D-\sqrt{2 D^{2}-1}-\frac{\sigma}{3} D^{1 / 3}
$$

This is the value of the linewidth enhancement factor commonly measured in Fabry-Pérot-type edge emitters, which tend to operate in the vicinity of the gain peak due to its intrinsic multilongitudinal mode character. We observe (see Fig. 5) a monotonic increase of $\alpha_{p}$ with carrier density, and also that the larger the band-gap renormalization, the smaller the value of $\alpha_{p}$. Accordingly, in Fabry-Pérot-type edge emitters, the $\alpha$ factor increases for increasing threshold gains, since in this case, larger carrier densities are required for the threshold being reached. In connection with the discussion on the differential gain, it turns out that operating the system close to transparency is doubly beneficial, since one then achieves a larger differential gain (which allows for higher modulation bandwidths) and a reduced $\alpha$ factor.

For finite temperatures, I could not work out an analytical approximation for the refractive index change. The reason is that the Lorentzian line shape implies that the real part of each transition has slowly decaying tails that have to be convolved with the Fermi-Dirac function in order to calculate the spectrum of the refractive index change. However, the refractive index spectrum can be numerically determined ei-

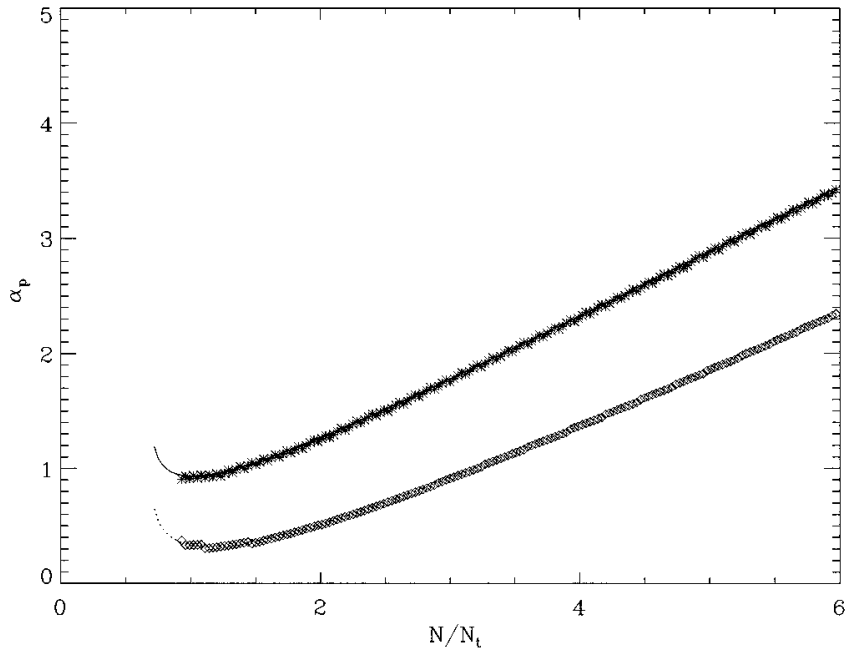

FIG. 5. Linewidth enhancement factor at the gain peak, $\alpha_{p}$, as a function of the normalized carrier density $N / N_{t}$. The symbols are obtained numerically from the electrical susceptibility, while the solid line corresponds to Eq. ( 18).

ther by direct integration of Eq. (2) or by the KramersKronig transformation of Eq. (15).

\section{STEADY-STATE OPERATION OF A SINGLE-MODE LASER}

A first assessment of the capabilities of the model developed in the previous section for the gain and refraction index can be achieved by analyzing its predictions for a singlemode laser, i.e., a single transverse-mode distributed feedback laser (DFB) or a single-transverse mode vertical-cavity surface-emitting laser (VCSEL). In the slowly varying amplitude approximation, the condition for $\mathrm{cw}$ operation reads

$$
\begin{aligned}
-i \delta \omega E= & \left\{\frac{c}{2 n_{g}}\left[-\alpha_{\mathrm{tot}}+i \Gamma \frac{\omega_{0}}{c n} \chi\left(\omega_{0}+\delta \omega, N\right)\right]\right. \\
& \left.-i\left(\omega_{c}-\omega_{0}\right)\right\} E, \\
0=\mathcal{C}-A N- & B N^{2}+|E|^{2} \frac{c}{n_{g}} \frac{\omega_{0}}{c n} \operatorname{Im}\left[\chi\left(\omega_{0}+\delta \omega, N\right)\right],
\end{aligned}
$$

where $E$ is the modal amplitude of the field normalized such that $|E|^{2}$ corresponds to photon density, $N$ is the carrier density in the active region, and $\chi(\omega, N)$ is the electrical susceptibility of the active region. $\mathcal{C}=I / e V$ is the carrier density injected per unit time into the active region whose volume is $V$ (I neglect leakage current), $A$ is the nonradiative recombination rate, $B$ is the bimolecular recombination coefficient due to spontaneous emission, and I have neglected Auger recombination due to the low-temperature approximation made. $\omega_{c}$ is the optical frequency corresponding to the cavity resonance, and $\delta \omega$ is the frequency deviation of the optical field from $\omega_{0}$, which I take as the carrier frequency. $n\left(n_{g}\right)$ is the effective refractive (group) index at $\omega_{0}$, and $\Gamma$ is the field confinement factor to the active region. The total losses in Eq. (19) are given by 


$$
\alpha_{\text {tot }}=\alpha_{\text {int }}-\frac{1}{L} \ln \left(r_{1} r_{2}\right),
$$

thus including both the internal losses $\left(\alpha_{\text {int }}\right)$ and the cavity losses $\alpha_{\text {end }}=L^{-1} \ln \left(r_{1} r_{2}\right)$ due to the facet reflectivities, $r_{1}$ and $r_{2}$, distributed over the effective length of the optical cavity, $L$ (for DFB lasers, $r_{1}$ and $r_{2}$ are the effective reflectivities for the left- and right-going waves). It is worth noting that in most single-mode devices at least one of the reflectivities is frequency dependent due to the presence of a grating either inside (DFB) or at the edges (the distributed Bragg reflectors of a VCSEL) of the cavity; however, I shall consider that in a relatively broad frequency range around the cavity resonance the facet reflectivities can be taken as constant (i.e., the lasing mode does not approach the edges of the stop band).

In order to reduce the number of parameters, I rescale Eqs. (19) $-(20)$ to

$$
\begin{gathered}
\delta \Omega=-\frac{\varepsilon}{2} \operatorname{Re}[a f(\delta \Omega, D)]+\Delta, \\
0=1+a \operatorname{Im}[f(\delta \Omega, D)], \\
P=J-r D-D^{2},
\end{gathered}
$$

where $\quad D=N / N_{t}, \quad \delta \Omega=\delta \omega / \gamma, \quad \Delta=\left(\omega_{c}-\omega_{0}\right) / \gamma, \quad a$ $=\Gamma \chi_{0} \omega_{0} /\left(c n \alpha_{\text {tot }}\right), \varepsilon=c \alpha_{\text {tot }} /\left(n_{g} \gamma\right), r=A / B N_{t}$ and

$$
P=|E|^{2} \frac{c}{n_{g}} \frac{\omega_{0}}{c n} \frac{\chi_{0}}{B N_{t}^{2}}
$$

Finally,

$$
f(\theta, D)=2 \ln \left(1-\frac{D}{\theta+\sigma D^{1 / 3}+i}\right)-\ln \left(1-\frac{b}{\theta+\sigma D^{1 / 3}+i}\right),
$$

where $b$ has been defined just following Eq. (6). The first and second equations determine the lasing frequency $\delta \Omega$ and the threshold carrier density for this frequency, which then determines the output power $P$ for a given current injection $J$. It is worth noticing that the only role of $r$ is to redefine the threshold current (and hence the output power), but it does not affect the threshold carrier density. In high-quality lasers that are free from structural defects in the active region, bimolecular recombination dominates over nonradiative recombination. Hence for the sake of simplicity I will simply consider $r=0$, because it will merely shift down the output power for a given current density.

By using Eq. (6) for the electrical susceptibility of the single quantum well, we can numerically solve the above set of equations by taking as a control parameter the normalized detuning between the cavity resonance and the nominal band-gap frequency.

Figure 6 shows the dependence of the threshold current on the lasing frequency for different values of the modal gain $a$. All curves are asymmetric around the minimum threshold, with smoother tails towards the blue side of the minimum threshold frequency than towards its red side, in agreement with the experimental results for VCSELs with different cavity resonances [30] or in tunable external-cavity lasers [31].

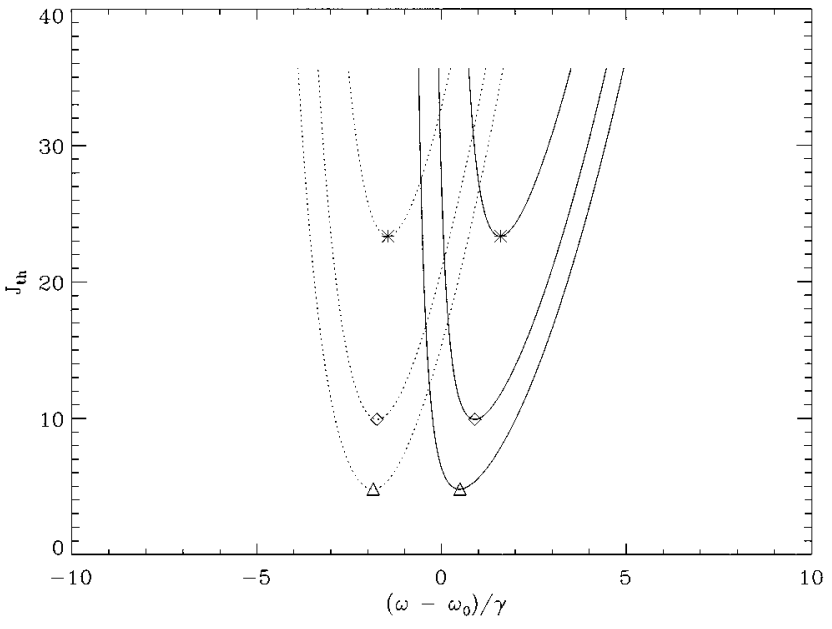

FIG. 6. Normalized threshold current density $J_{t} h$ as a function of the lasing frequency for $\sigma=0.2$ (solid lines) and $\sigma=2$ (dashed lines) and different values of the modal gain $a=1$ (triangle), 2/3 (diamond), and 1/2 (star).

However, the asymmetry decreases for increasing band-gap renormalization, since it contributes additional gain on the red side of the minimum threshold. The asymmetry of these curves will be barely the same for $r \neq 0$, although all the curves will be shifted upwards due to the increase in threshold current density associated with the increased carrier recombination rate.

The asymmetry of the threshold curve around its minimum is clearly noticeable in Fig. 7, which shows the $\mathrm{cw}$ output power of the laser versus lasing frequency (stars) and the $\alpha$ factor at the operation point (diamonds) for two different values of the modal gain. The injection currents have been chosen to yield the same maximum output power. It can be observed that the asymmetry in the output power is more noticeable for small $\sigma$ [cases (a) and (c)] since, as already
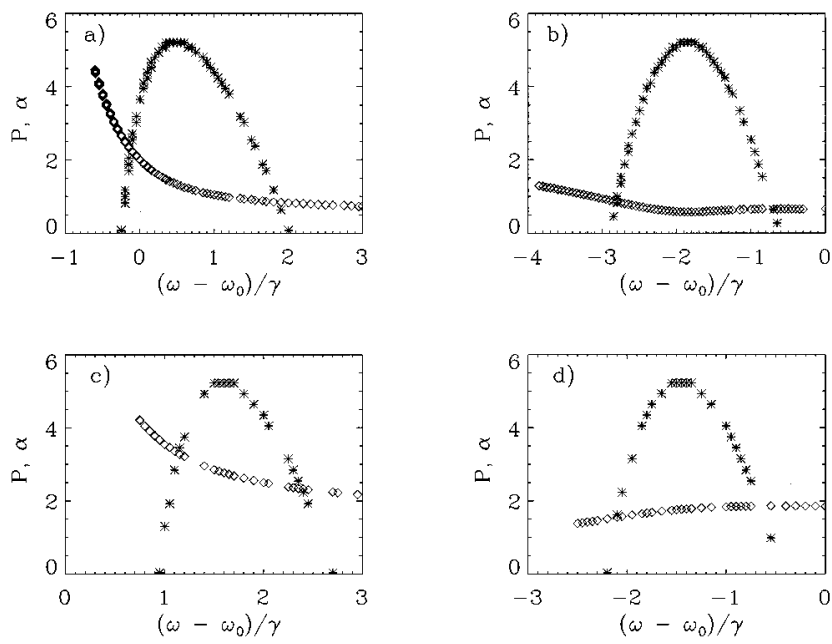

FIG. 7. Normalized output power $P$ (stars) and $\alpha$ factor (diamonds) for $\mathrm{cw}$ operation of the laser as a function of the lasing frequency: (a) $\sigma=0.2$ and $a=1$, (b) $\sigma=2$ and $a=1$, (c) $\sigma=0.2$ and $a=1 / 2$, and (d) $\sigma=2$ and $a=1 / 2$. The pump current in cases (a) and (b) is $J=10$, while in cases (c) and (d) it is $J=28.55$, chosen such that the normalized output power is the same in all cases. 


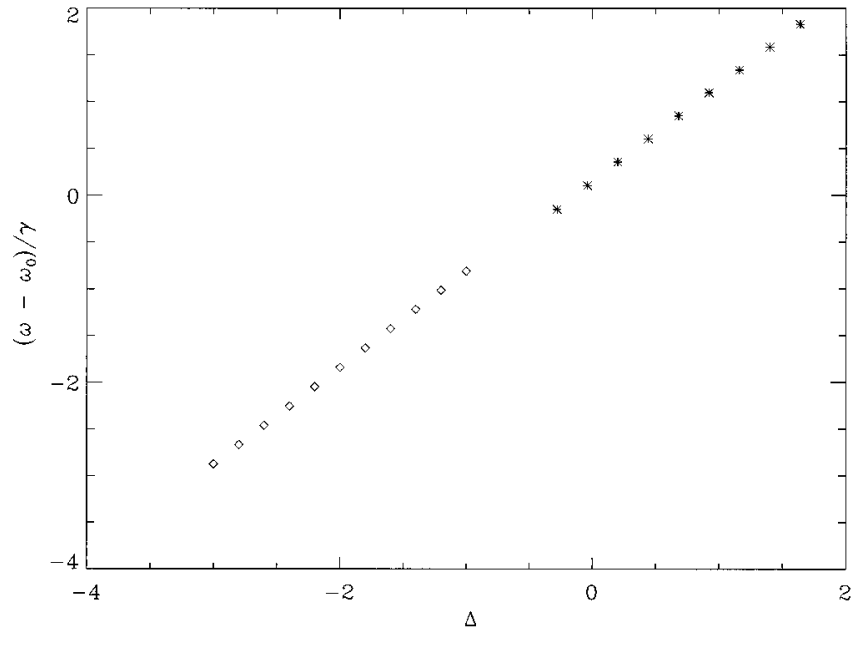

FIG. 8. Normalized lasing frequency $\left(\omega-\omega_{0}\right) / \gamma$ as a function of cavity detuning $\Delta=\left(\omega_{c}-\omega_{0}\right) / \gamma$, for modal gain $a=1$ and $J$ $=10$. Stars correspond to $\sigma=0.2$, and diamonds to $\sigma=2$.

commented, larger band-gap renormalizations contribute additional gain to low frequencies. In addition, we can also see that the asymmetry is stronger for high modal gain [case (a)] due to the reduced band-gap shrinkage that occurs in this case.

One can also see the dramatic influence that the lasing frequency and modal gain have on the $\alpha$ factor at the operation point: reduced threshold gains [cases (c) and (d)] yield larger $\alpha$ factors at the operation point, while increased bandgap renormalization coefficients [cases (b) and (d)] lead to much reduced $\alpha$ factors at the operation point. In addition, it can also be seen that, for low band-gap renormalization parameter, a noticeable reduction in the $\alpha$ factor can be achieved by operating the laser on the blue side of the minimum threshold [cases (a) and (c)], in agreement with the experimental results in $[27,28]$. However, this effect vanishes or may be even reversed when the system has large band-gap renormalization coefficient [see cases (b) and (d)].

Finally, in Fig. 8 I have plotted the operation frequency as a function of detuning for $a=1$. It can be seen that the operation frequency is always a little bit higher than the cavity mode, the reason being the positive slope of the real part of the susceptibility in the region of positive material gain.

It is worth noting, in connection with Figs. 7 and 8, that the above results have been obtained by varying only the cavity detuning, the other parameters remaining constant. The inclusion of gain and dispersion spectra through the electrical susceptibility of the QW allows one to reproduce the variation of both the output power and the $\alpha$ factor as a function of cavity detuning.

\section{SUMMARY}

In summary, I have presented an analytical expression for the optical susceptibility of a QW at low temperatures from which several properties of the gain, differential gain, refraction index, and $\alpha$ factor of the active medium can be found. The results are in qualitative agreement with those obtained from more fundamental, microscopic theories, and also with several experimental observations. For fixed threshold gain, the threshold carrier density varies with detuning following the spectral gain curve, and so do the output power and linewidth enhancement factor at the operation point. Therefore, the model described improves the standard RE description by incorporating the gain and dispersion spectra of the QW laser. The model can be useful for analyzing the lasing properties of multimode and inhomogeneous devices without requiring one to use a fully microscopic theory, hence speeding up computer simulations. An analytical approximation for the gain at finite temperature has also been presented, although the refractive index spectrum requires a numerical evaluation. Generalization of this work to study the dynamics of such devices is in progress.

\section{ACKNOWLEDGMENTS}

This work has been supported by Comision Interministerial de Ciencia y Tecnologia (CICYT, Spain) Project No. TIC95-0563-CO5. I also acknowledge financial support from the Human Capital and Mobility Program of the European Union, Contract No. FMRX-CT96-0066. Finally, I thank Professors M. San Miguel, J. R. Tredicce, P. Colet, E. Hernández-García, and N. B. Abraham for their support, useful discussions, and a critical reading of this manuscript.
[1] See, e.g., M. Sargent III, M. Scully and W. Lamb, Laser Physics (Addison-Wesley, Reading, MA, 1977).

[2] H. Statz and G. A. deMars, in Quantum Electronics, edited by C. H. Townes (Columbia University Press, New York, 1960); C. L. Tang, H. Statz, and G. deMars, J. Appl. Phys. 34, 2289 (1963).

[3] A. Yariv, Optical Electronics (Holt, Rinehart and Winston, Philadelphia, 1991).

[4] A. E. Siegman, Lasers (University Science Books, Mill Valley, CA, 1986).

[5] K. Shimoda, Introduction to Laser Physics, Springer Series in Optical Sciences Vol. 44 (Springer-Verlag, Berlin, 1986).

[6] G. P. Agrawal and N. K. Dutta, Long-Wavelength Semiconductor Lasers (Van Nostrand-Reinhold, New York, 1986).
[7] B. Mroziewicz, M. Bugajski, and W. Nakwaski, Physics of Semiconductor Lasers (North-Holland, Amsterdam, 1991).

[8] L. A. Coldren and S. W. Corzine, Diode Lasers and Photonic Integrated Circuits (Wiley, New York, 1995).

[9] Y. Zao, J. S. Osinski, P. Grodzinski, P. D. Dapkus, W. C. Rideout, W. F. Sharfin, J. Schlafer, and F. D. Crawford, IEEE J. Quantum Electron. 29, 1565 (1993); S. L. Chuang, J. O'Gorman, and A. F. J. Levi, ibid. 29, 1631 (1993); Z.-M. Li, M. Dion, Y. Zhu, J. Wang, M. Davies, and S. P. McAlister, ibid. 30, 538 (1994); D. A. Ackerman, G. E. Shtengel, M. S. Hybertsen, P. A. Morton, , T. Tanbum-Ek, and R. A. Logan, IEEE J. Sel. Top. Quantum Electron. 1, 250 (1995).

[10] C. H. Henry, IEEE J. Quantum Electron. 18, 259 (1982).

[11] F. Stern, J. Appl. Phys. 47, 5382 (1976); D. S. Citrin and Y.-C. 
Chang, IEEE J. Quantum Electron. 29, 97 (1993); T. Yamanaka, Y. Yoshikuni, K. Yokoyama, W. Lui, and S. Seki, ibid. 29, 1609 (1993); W. L. Li, Y. K. Su, and D. H. Jaw, ibid. 33, 416 (1997).

[12] P. M. Enders, IEEE J. Quantum Electron. 33, 580 (1997).

[13] H. Haug and S. Schmitt-Rink, Prog. Quantum Electron. 9, 3 (1984); M. Lindberg and S. W. Koch, Phys. Rev. B 38, 3342 (1988); H. Haug and S. W. Koch, ibid. 39, 1887 (1989).

[14] W. W. Chow, S. W. Koch, and M. Sargent III, SemiconductorLaser Physics (Springer-Verlag, Berlin, 1994).

[15] O. Hess and T. Kuhn, Phys. Rev. A 54, 3347 (1996); 54, 3360 (1996).

[16] T. Makino, IEEE J. Quantum Electron. 32, 493 (1996).

[17] C. M. Bowden and G. P. Agrawal, Opt. Commun. 100, 147 (1993); J. Yao, G. P. Agrawal, P. Gallion, and C. M. Bowden, ibid. 119, 246 (1995).

[18] S. Balle, Opt. Commun. 119, 227 (1995).

[19] M. Homar, S. Balle, and M. San Miguel, Opt. Commun. 131, 380 (1996).

[20] R. Binder, D. Scott, A. E. Paul, M. Lindberg, K. Henneberger, and S. W. Koch, Phys. Rev. B 45, 1107 (1992).

[21] H. Haug and S. W. Koch, Quantum Theory of the Optical and Electronic Properties of Semiconductors, 2nd ed. (World Sci- entific, Singapore, 1993), Fig. 21.1 on p. 413; C. Y. Tsai, L. F. Eastman, and Y. H. Lo, Appl. Phys. Lett. 63, 3408 (1993); see also [15], Figs. 1(d)-1(f) on p. 3353.

[22] M. Asada, in Quantum Well Lasers, edited by P. S. Zory, Jr. (Academic Press, San Diego, 1993).

[23] A. Tomita and A. Suzuki, IEEE J. Quantum Electron. 23, 1155 (1987).

[24] D.A. Kleinman and R. C. Miller, Phys. Rev. B 32, 2266 (1985).

[25] D. J. Bossert and D. Gallant, IEEE Photonics Technol. Lett. 8, 322 (1996).

[26] A. Niwa, T. Ohtoshi, and T. Kuroda, IEEE J. Sel. Top. Quantum Electron. 1, 211 (1995).

[27] A. Schöenfelder, S. Weisser, J. D. Ralston, and J. Rosenzweig, IEEE J. Sel. Top. Quantum Electron. 6, 322 (1994).

[28] M. Hofmann, M. Koch, H.-J. Heinrich, G. Weiser, J. Feldmann, W. Elsässer, E. O. Gobel, W. W. Chow, and S. W. Koch, IEE Proc.: Optoelectron. 141, 127 (1994).

[29] W. L. Li, Y. K. Su, and D. H. Jaw, IEEE J. Quantum Electron. 33, 416 (1997).

[30] T. E. Sale, Vertical Cavity Surface Emitting Lasers (Wiley, New York, 1995).

[31] J. O’Gorman and A. F. J. Levi, Appl. Phys. Lett. 62, 2009 (1993). 